\title{
Análisis cuantitativo de la percepción sobre la discriminación en la población con orientación sexual e identidad de género diversas y la población de negritudes en los estudiantes universitarios
}

\section{Quantitative analysis of the perception of discrimination in the population with diverse sexual orientation and gender identity and the black population among university students}

\author{
Rocío de Belén Contreras-Manrique ${ }^{\mathrm{a}}$, Tatiana Valentina Ovalle-Lizcano ${ }^{\mathrm{b}}$, Liliana Contreras-Manri- \\ que $^{c}$, Diego Luis Coronel-Peñuela ${ }^{\mathrm{d}}$ \\ aMagister en Prácticas Pedagógicas, rociodebelen@unipamplona.edu.co, https://orcid.org/0000-0002-4434-0408, Universidad de Pamplona, Villa Rosario, Colombia \\ bAbogada en formación, tatiana.ovalle@unipamplona.edu.co, https://orcid.org/0000-0003-3643-8584, Universidad de Pamplona, Villa del Rosario, Colombia \\ 'Magister en Orientación, lilianacontrerasmanrique@yahoo.com.mx, https://orcid.org/0000-0002-8586-2093, Universidad de Pamplona, Villa del Rosario, Colombia \\ dMagister en Derecho, dlcp11@hotmail.com, https://orcid.org/0000-0003-0580-1109, Universidad de Pamplona, Villa del Rosario, Colombia
}

Forma de citar: Contreras-Manrique, L., Coronel-Peñuela, D. L., Contreras-Manrique, R. de B.., \& Ovalle-Lizcano, T. V. (2020). Análisis cuantitativo de la percepción sobre la discriminación en la población con orientación sexual e identidad de género diversas y la población de negritudes en los estudiantes universitarios. Eco Matemático, 11(2), $66-80$

Recibido: 4 Abril 2020

Aceptado: 19 Mayo 2020

\section{Palabras clave \\ Discriminación; \\ LGTBI; \\ Orientación sexual; \\ Identidad de género \\ diverso; \\ Negritudes}

*Autor para correspondencia:rociodebelen@unipamplona.edu.co Constitución.
Resumen:Analiza la percepción sobre la discriminación en la población con orientación sexual e identidad de género diverso y la población de negritudes en los estudiantes universitarios. Metodología/Enfoque. Cuantitativa, diseño de la investigación, descriptivo, muestra 100 universitario. Resultados/Hallazgos Un mayor porcentaje de los estudiantes encuestados eligieron que no se puede obligar o convencer a una persona para que dejen la comunidad LGTBI; por lo tanto, aceptan la posición de los estudiantes que pertenecen a esta comunidad $y$, por consiguiente, en un porcentaje mínimo, se encuentran con sentimientos de tristeza y baja autoestima. De tal manera, Un 64\% en los encuestados han visto rechazo o discriminación en la identidad de género diversas por parte de sus compañeros universitarios. Por último, un $73 \%$ en los encuestados, las personas de color o la población de negritudes, si son discriminadas. Discusión/Conclusiones/Contribuciones. El papel de la comunidad educativa (padres y madres de familia, estudiantes, docentes, profesionales de psicología, enfermera, médico y trabajo social, es convivir diariamente con la diversidad de género y la comunidad negra con valores, en especial el respeto, la diferencia, la igualdad, los derechos humanos y dando cumplimiento a lo que establece la 


\section{Keywords}

Discrimination;

LGTBI;

Sexual orientation;

Diverse gender identity;

Negritudes

\begin{abstract}
Analyzes the perception of discrimination in the population with diverse sexual orientation and gender identity and the population of blackness in university students. Methodology / Approach. Quantitative, research design, descriptive, sample 100 university students. Results / Findings A higher percentage of the surveyed students chose that it is not possible to force or convince a person to leave the LGTBI community; therefore, they accept the position of the students who belong to this community and, consequently, in a minimal percentage, they find feelings of sadness and low self-esteem. In this way, $64 \%$ of those surveyed have seen rejection or discrimination in the diverse gender identity on the part of their university companions. Lastly, 73\% of respondents, people of color or the black population, if they are discriminated against. Discussion / Conclusions / Contributions. The role of the educational community (parents, students, teachers, psychology professionals, nurse, doctor and social work, is to live daily with gender diversity and the black community with values, especially respect, difference, equality, human rights and in compliance with what the Constitution establishes.
\end{abstract}

\section{Introducción}

Actualmente existe en la sociedad la discriminación en la población con orientación sexual e identidad de género diversas y la población de negritudes entre los jóvenes universitarios. Por lo tanto, para los autores es relevante el tema en el análisis y descripción de la problemática en las percepciones de la sociedad con respecto a la población diversa de género y la población de negritudes.

La corte interamericana de Derechos Humanos. Derechos de las personas LGTBI / Corte Interamericana de Derechos Humanos. -- San José, C.R.: Corte IDH. (2018). La inclusión de la orientación sexual y la identidad de género como categorías de discriminación están prohibidas, el Tribunal Europeo de derechos humanos ha señalado que la orientación sexual y la identidad de género se pueden entender como incluidas dentro de la categoría "otra condición" mencionada en el artículo 14 del Convenio Europeo para la Protección de los derechos humanos y de las Libertades Fundamentales (en adelante "Convenio Europeo"), donde hace énfasis la prohibición de los tratos discriminatorios. En particular, en el caso Salgueiro da Silva Mouta Vs. Portugal, el Tribunal Europeo concluyó que la orientación sexual es un concepto que se encuentra cubierto por el artículo 14 del Convenio Europeo.

Resalto el caso Clift Vs. Reino Unido, el Tribunal Europeo en la cual reiteró que la orientación sexual, como una de las categorías que puede ser incluida bajo "otra condición", que se consideran como características personales en el sentido que son innatas o inherentes a la persona. Por tal motivo, el consejo de Europa ha adoptado una serie de textos encaminados a combatir la discriminación basada en la

orientación sexual y en una menor medida en la identidad de género; siendo una responsabilidad en garantías de respeto, dignidad que se establece en el artículo 1.1 de la convención Americana, que se encuentra en el artículo 29 de la Convención de Viena sobre el Derecho de los Tratados, las Resoluciones de la Asamblea General de la OEA, y los organismos de Naciones Unidas (supra párrs. 71 a 76), la Corte Interamericana deja establecido que la orientación sexual y la identidad de género, 
así como la expresión de género son categorías protegidas por la Convención. cualquier norma, acto o práctica discriminatoria basada en la orientación sexual, identidad de género o expresión de género de la persona con los derechos de una persona a partir de su orientación sexual, su identidad de género y/o su expresión de género.

A continuación, en el principio de Yogyakarta. (2007). Menciona, que todas las personas tienen derecho al disfrute de todos los derechos humanos, sin discriminación por motivos de orientación sexual o identidad de género. De tal manera, tienen derecho a ser iguales ante la ley y tienen derecho a igual protección por parte de la ley, sin ninguna de las discriminaciones. En la cual se resalta, que la ley prohibirá toda discriminación de esta clase y garantizará a todas las personas protección igual y efectiva contra cualquier forma de discriminación por motivos de orientación sexual o identidad de género, raza, edad, religión, discapacidad, estado de salud y condición económica.

En cuanto a los derechos de la población negra o afrocolombiana, Ley No. 1833 del 2017 y crea la comisión legal para la protección de los derechos de las comunidades negras o población afrocolombiana del congreso de la república de Colombia. Con el fin de asegurar la protección de los derechos colectivos e individuales en el mejoramiento de sus condiciones y calidad de vida a partir de la gestión legislativa, institucional, organizativa, $\mathrm{y}$ el control político que realicen los congresistas afrocolombianos a través de esta comisión Legal. Por lo tanto, la comisión, de corte pluralista, étnica y democrática, tiene como objetivo trabajar coordinadamente para la superación de las grandes desigualdades que separan a los afrocolombianos del resto de la sociedad: propendiendo por el respeto y garantía de la diversidad étnica y cultural de la nación; la defensa de su patrimonio; la generación de espacios y canales efectivos de participación y visibilización de la población en el contexto local, nacional e internacional.
En la investigación, se destacan algunos autores como soporte a la temática estudiada.

El autor Bimbi (2017) "Después

de salir del armario interior, llega el momento de entender que no es malo, que ser gay es tan normal y natural como ser hetero y que está todo bien. Transformar la vergüenza en orgullo es algo que no todos consiguen, pero es imprescindible para llevar una vida sana y feliz, manteniendo la autoestima y con igualdad de derecho como un heterosexual" (p.14).

En la investigación se tiene en cuenta, la teoría de Rodríguez. (2015) ya que contribuye con el concepto discriminación, direccionándolo como una conducta, culturalmente fundada, de desprecio contra una persona o grupos de personas, sobre la ideología de un prejuicio negativo, o un estigma relacionado con una desventaja inmerecida (p.23).

En este mismo sentido Lambda Legal (2013), contribuyo teóricamente con las iniciales LGTB, que hacen referencia a lesbianas, gay, bisexuales, como una forma inclusiva a todos los individuos (p.1); por lo tanto, en otra teoría que cabe resaltar es la teoría general de sistemas fue concebida por Ludwig von Bertalanffy en la década de (1940); proporcionando un marco teórico y práctico a las ciencias naturales y sociales.

En la vida universitaria, se ha tenido avances en la aceptación hacia la comunidad LGBT, debido a esto; aún existe el rechazo hacia esta comunidad en los diferentes ámbitos sociales perjudicando, así, a los educandos en su satisfacción de vida; por este motivo se presenta deserción académica.

Los derechos igualitarios de los miembros de la población con orientación sexual e identidad de género diversos se encuentran en un constante desarrollo, y aplicabilidad dentro de los diferentes ámbitos educativos; debido a las diferentes normatividades jurídicas que sancionan cualquier acto discriminatorio en contra de los miembros de 
esta comunidad. No obstante, a la desagregación en las universidades.

Según Francisco Antar Martínez Guzmán (2017), señaló la problemática de violencia y discriminación contra la comunidad lésbico, gay, bisexual, transgénero (LGBT) y hay más apertura institucional para generar normativas que coadyuven a combatir este problema social; también, Resaltó "La violencia y la discriminación se han desplazado hacia formas más sutiles y cotidianas, menos evidentes en su forma de operar. Sigue habiendo todavía prejuicio, discriminación y violencia simbólica, solo que se da a través de medios más velados y menos visibles, que ocurren en interacciones cotidianas a nivel microsocial.

Es precisamente la Corte Constitucional la que ha avanzado para los derechos de las personas miembros de la población con orientación sexual e identidad de género diversas.

En el caso colombiano, Cabe señalar que la violación de los derechos de las personas LGBTI y el incumplimiento de las obligaciones de regulación del Congreso han sido enfrentados por los jueces de la jurisdicción constitucional mediante mecanismos adicionales a los que ofrece la inconstitucionalidad por omisión, como la emisión de sentencias condicionadas, la emisión de exhortos y la emisión de órdenes de regulación a las autoridades administrativas, que son todo un avance por ahora dispuesto respecto al derecho a morir dignamente (Corte Constitucional, 2014), el derecho a la objeción de conciencia al servicio militar (Corte Constitucional, 2014) y los derechos de las personas transexuales, a propósito de las estructuras de dominio sobre el cuerpo que propician la violación permanente de los derechos de las personas LGBTI.

Bazán (2014). El derecho a la igualdad establecido en el Artículo 13 de la Constitución no ha sido desarrollado por el Congreso de la República en el caso de los derechos de las personas LGBTI y de los transexuales, en una situación en donde no solo ha habido incuria y omisión, sino verdadera resistencia. En segundo lugar, resulta evidente que la falta de desarrollo legislativo del derecho a la igualdad se ha mantenido durante veinticinco años, a pesar de los esfuerzos hechos por la Corte Constitucional: durante la década del noventa, comenzó a reconocer los derechos de esas personas; en la de 2000 reconoció y protegió expresamente los derechos de las parejas del mismo sexo y durante la década del diez viene extendiendo el reconocimiento y la protección de esos derechos a los otros miembros de la comunidad LGBTI. Quinche Ramírez, Manuel Fernando. (2016). Violencias, omisiones y estructuras que enfrentan las personas LGTBI. Interpreto, y diagnóstico contenido en el Informe de la Comisión Interamericana denominado "Violencia contra personas LGBTI". Desde allí se explicitan otras formas de violencia, entre ellas, la violencia institucional que se origina en el incumplimiento de las obligaciones de respeto y garantía sobre los derechos de esas personas. Además, se trabaja el tema de las omisiones legislativas y convencionales e identifica algunas estructuras tradicionales de dominio que impiden el reconocimiento y la protección de los derechos de esa comunidad. Las conclusiones del Informe de la Comisión Interamericana son tan obvias como preocupantes, en el sentido de que evidencian diversas formas de violencia generalizada en contra de esas personas, que tiene en la base una historia local de intolerancia sobre grupos sociales discriminados. Desde esa perspectiva, el Informe concluye:

[...] que el contexto generalizado de discriminación social e intolerancia respecto de esta diversidad, aunado a la ausencia de investigaciones efectivas, y la falta de un abordaje diferenciado para prevenir, investigar, juzgar, sancionar y reparar los crímenes cometidos contra personas LGBTI, son elementos que conducen a que se condone y tolere esta violencia, lo que resulta en impunidad y repetición (Comisión IDH, 2015, párr. 508). 


\section{Objetivos}

\section{Objetivo General}

Analizar la percepción sobre la discriminación en la población con orientación sexual e identidad de género diversas y la población de negritudes en los estudiantes de la Universidad de Pamplona, de Villa del Rosario a través de una encuesta.

\section{Objetivos Específicos}

Describir la percepción sobre la discriminación en la población con orientación sexual e identidad de género diversas y la población de negritudes en los estudiantes de la Universidad de Pamplona, de Villa del Rosario a través de una encuesta.

En el desarrollo del estudio se resaltó algunos autores a nivel internacional, nacional y regional como apoyo en la investigación. A continuación, se citan los autores que realizaron estudios sobre La discriminación de La población con Orientación Sexual e identidad de género Diversas y la población negra

Rodríguez Otero, Luis-Manuel; Ávila Zárate, Lydia del Carmen. (2019). Actitudes hacia la diversidad. La discriminación y el estigma en estudiantes de trabajo social mexicanos, siendo los rasgos y las actitudes de las personas en las estructuras sociales que generan procesos, donde se jerarquiza de forma inferior a quienes se etiquetan bajo dichas características; siendo devaluados y estigmatizando a dicha población. Los resultados que la sexualidad y la etnia son los rasgos más susceptibles al estigma.

Rodríguez Otero, Luis-Manuel y Facal Fondo, Teresa. (2019). Imaginarios, mitos y actitudes frente a la bisexualidad en estudiantes de trabajo social. En un proceso socialmente como dicotomiza, estereotípica y jerarquiza a la población, se identificó la percepción social que poseen sobre la homosexualidad, la bisexualidad y la transexualidad. Es importante, que se considera la promoción de la igualdad y la diversidad para la construcción de los estigmas.

Martínez -Guzmán, Antar And IniguezRueda, Lupicinio. Prácticas discursivas y violencia simbólica hacia la comunidad LGBT en espacios universitarios (2017). La violencia simbólica dirigida hacia la comunidad lésbico, gay, bisexual y transgénero (LGBT). Por tal motivo, Explorando las formas de violencia simbólica hacia personas LGBT a través del discurso de estudiantes y docentes en el contexto universitario mexicano.

Pena Tosso. (2015). Homofobia en las aulas universitarias. Estudio la homofobia en el sistema educativo, también por el reconocimiento legal que las personas Lesbianas, Gays, Bisexuales y Transexuales han ido adquiriendo en los últimos tiempos, fruto de años de luchas y reivindicaciones. Los resultados se evidencia los aspectos más estudiados en relación con la homofobia en las aulas universitarias. finalmente, se discutió, la pertinencia de trabajar la diversidad afectivosexual y la prevención de la homofobia desde los centros universitarios, las diferentes maneras de hacerlo y el valor agregado que tiene el profesorado universitario en este aspecto relacionado con el sexismo, la atención a la diversidad, la equidad y la inclusión educativa.

Mancilla Alcazar, Adriana Michelle (2016). Percepción de las personas homosexuales sobre la aceptación al asumir públicamente su orientación sexual. El autor, identificó cuál es la percepción de las personas homosexuales sobre la aceptación al asumir públicamente su orientación sexual. Conclusión se arrojó, que las personas homosexuales sí presentan incertidumbre y temor al momento en aceptar públicamente su orientación sexual, en el ámbito familiar. En el ámbito social, es relevante la aprobación y en pocos casos las personas son discriminadas por sus iguales. Un tema demandante 
en la investigación fue la discriminación que se presenta en el área laboral, siendo causa de despidos, burlas, exclusión, entre otras.

Jaramillo J. E., Rincón Leal J. F., Rincón Leal O. L. (2020). Estudiantes migrantes en la adaptación de la educación colombiana el proceso de adaptación escolar colombiano con los estudiantes migrantes de la zona de frontera del corregimiento de la parada, identificar los factores que influyen en la inteligencia emocional en los estudiantes migrantes. La investigación resalta los factores que influyen en la inteligencia emocional como atención o percepción, comprensión y regulación del ser humano. Por consiguiente, se aplicó el cuestionario como herramienta de estudio la escala TMMS-24, de Salovey y Mayer, con un diseño descriptivo cuantitativo, muestra 100 migrantes. Por lo tanto, la educación colombiana tiene el compromiso de orientar a los educandos

Liscano Rivera Doria Constanza y Jurado de los Santos Pedro. (2016). Representaciones Sociales sobre las personas LGBTI en la universidad perspectivas del profesorado y alumnado. Barcelona. Esta investigación, sobre la percepción de la diversidad sexual y las representaciones sociales que se manifiesto en el contexto universitario formando parte de las prácticas del profesorado y alumnado frente a personas LGBTI. Se aplicó una metodología descriptiva ex -post-facto y enfoque cualitativo a través de un estudio de caso centrado en una Universidad. La manifestación de las sexualidades diversas, la construcción. Valorándose, los aspectos cognitivos, actitudinales y relacionales de la comunidad universitaria, una aprobación por una visión hegemónica heteronormativa, que busca ser transformada desde las prácticas poco homogéneas que limitan la percepción normalizadora del colectivo.

Por otro parte Samaniego \& Bermúdez (2015), realizaron su proyecto de grado Discriminación hacia jóvenes homosexuales en su entorno educativo. sobre las observaciones asociadas con la discriminación en el contexto educativo orientándolo así a estudiantes de Cuenca; La percepción de los entrevistados es que no hay conciencia en los pares en brindarles la adecuada información; también capacitar a los docentes en cuanto al tema de diversidad se refiere en el contexto universitario.

Piña \& Aguayo (2015), en su artículo Homofobia en estudiantes de México. El objetivo conocer la homofobia e inclusión de alumnos en tres universidades públicas mexicanas de las regiones centro, golfo y sureste. Se realizó las comparaciones de términos con concepto homosexual en 168 jóvenes. Se evaluó y se categorizo. En resumen, contribuye con nuestra investigación ya que nos permite la metodología de asociación de palabras, esclareció el verdadero impacto que genera en la población LGTB.

Jaramillo Gómez, Isabella y Moreno Méndez, Kelly Johana. (2018). Pontificia universidad javeriana Cali, Percepciones de estudiantes gays y lesbianas sobre diversidad sexual en contexto universitario. Se realizo la exploración en las percepciones de estudiantes gays y lesbianas sobre la diversidad sexual en el contexto universitario, estudio las percepciones sobre estigma, discriminación e inclusión de estudiantes gays y lesbianas en el contexto universitario, reconoce los discursos y escenarios identificados por los estudiantes en el contexto universitario frente al abordaje de la diversidad sexual.

Jiménez, Daniela y Quiroga Mery, Melissa (2016). Percepciones de la población LGBT sobre la formulación de la política pública local, Cali, Colombia. Presento los hallazgos de un proyecto investigativo entorno a la formulación de la política públicaparalapoblaciónLGBTlocalentreseptiembre y noviembre de 2013. indagaron las percepciones de la población LGBT sobre dicha política pública, la cual se ha desarrollado entre manifestaciones de resistencia de aquellos directamente afectados 
como de acciones institucionales para ejecutar su formulación.

Grajales Beatriz Elena, Ossa Henao Humberto, Klimenko Olena, Álvarez Posada José Luis. (2015). El artículo estudio la percepción de algunos residentes del Municipio de Itagüí con la orientación homosexual, al respecto del reconocimiento de la comunidad LGTBI a nivel social en Colombia". La población LGTBI son conscientes que aún falta mucho más por informar y mejorar el ambiente, sin verlo como un capricho, sino como una forma de ser incluidos socialmente.

Zambrano Guerrero, C., Hernández Pasichaná, P., \& Guerrero Montero, A. (2019). Proceso de reconocimiento de la orientación sexual homosexual en estudiantes de una universidad pública: Proceso de reconocimiento de la orientación sexual homosexual en estudiantes de una universidad pública. Analizaron con perspectiva propositiva el proceso de reconocimiento de la orientación sexual homosexual en estudiantes gays y lesbianas de una universidad pública de la ciudad de Pasto. Resultados: las dificultades a lo largo de este proceso de reconocimiento, como prejuicios, estereotipos y homofobia a nivel familiar, social, lo que conlleva al ocultamiento de la orientación. Se reconoció la importancia de las redes de apoyo como la familia y amigos, como base fundamental para el reconocimiento y aceptación de la homosexualidad. Es así, se proponen estrategias para fortalecer el proceso de reconocimiento de la orientación sexual a partir de ideas propuestas por los mismos participantes, entre las que se destacan: el empoderamiento homosexual, fortalecimiento de las redes de apoyo y la educación sobre la diversidad sexual. Se resalta la trascendencia de la parte social y familiar, y se generan posturas críticas enfatizadas en la aprobación del otro.

Cáceres Carvajal, M. O., Santiago, M.C. \& Rincón-Leal, O. L. (2019). Los rasgos de personalidad influyen en los jóvenes universitarios ya sea en su medio ambiente, particularmente en los procesos de socialización con niveles de ansiedad, sentimientos de tristeza o trastorno como la depresión, agresividad que afectan al estudiante en su proceso de aprendizaje e interacción con su comunidad educativa y en la formación profesional. Esta investigación se basó con el teórico de Erik Erickson, teniendo en cuenta las etapas en el desarrollo de la personalidad y haciendo énfasis en la etapa V (Identidad frente a Confusión de Roles), etapa VI (Intimidad frente a Aislamiento) donde se logró dar la importancia, que es base fundamental para entender el proceso del desarrollo humano, permitiendo al estudiante interactuar de manera flexible, armoniosa en el medio que lo rodea.

Méndez, Kelly Johana. (2018). Pontificia universidad javeriana Cali, Percepciones de estudiantes gays y lesbianas sobre diversidad sexual en contexto universitario. Se exploro las percepciones de estudiantes gays y lesbianas sobre la diversidad sexual en el contexto universitario, identificándose las concepciones de ser gay y lesbiana en el contexto universitario; indago percepciones sobre estigma, discriminación e inclusión de estudiantes gays y lesbianas en el contexto universitario, se reconoce los discursos y escenarios identificados por los estudiantes en el contexto universitario frente al abordaje de la diversidad sexual.

Gómez Blanco, Carmen Ginary. (2018). Diseño de una estrategia comunicativa para la inclusión social y el fomento de las prácticas culturales de la comunidad LGTBI en la universidad Francisco de Paula Santander Ocaña. La autora, realizo un diagnóstico situacional sobre la comunidad LGBTI dentro de la universidad y creo una cartilla con el nombre estrategia de comunicación participativa 'diverso', en la que se pueden observar tres fases en donde se describen las actividades competentes para fomentar la inclusión social y las prácticas culturales de la comunidad LGBTI en la universidad Francisco de Paula Santander Ocaña. 
Actualmente, la comunidad LGBTI habla de inclusión a través de actividades culturales, que involucran tanto a su comunidad, como a la población en general. Así como lo asegura Caicedo (2018), al afirmar que: cuarenta y nueve años después de que se originara la conmemoración del Día del Orgullo de la comunidad LGBTI (lesbianas, gais, bisexuales, transexuales e intersexuales), este sector de la población ha logrado abrir espacios de inclusión en Cúcuta a través de cine rosa, marcha del orgullo gay y la pasarela T. (p. 1).

La percepción depende del punto de vista que posea cada una de las personas sobre una situación dada en un momento específico. Como indica Melton (2007), la realidad de las cosas se entiende por el entorno en el que se encuentra una persona, la cual llama "perceptor". Esta persona percibe el entorno y le brinda un significado que depende de la situación perceptiva, y llega así a una conclusión válida con las interpretaciones y diferentes experiencias pasadas que se complementan para crear una realidad.

\section{Teoría}

Teoría Queer de Butler (2009). La teoría queer cuestiona la existencia de categorías tales como las de hombre, mujer, heterosexual, homosexual, bisexual, y lesbiana, entre otras, cuestionando ese concepto prescriptivo de "naturaleza". Según este enfoque, todas ellas son construcciones sociales y su imposición, bajo la responsabilidad de la naturaleza, encubre una ética disciplinaria. Esto implica que no son de orden natural o divino, sino que surgen como resultado de procesos de índole social.

Natalio Kisnerman (1990). Hablo sobre las causas-efectos de los problemas sociales y de lograr que los hombres asuman una acción organizada, tanto preventiva como transformadora, que los supere y así se integren las personas de LGTBI y las comunidades negras en las diferentes etapas del grupo entre ellas se encuentran; la etapa de formación o aparente grupo, Etapa de conflicto,
Etapa de organización, Etapa de integración y Etapa de declinación o muerte del grupo.

\section{Diseño Metodológico}

La investigación que se desarrolló desde el enfoque cuantitativo. Usando un diseño explicativo y muestreo intencional, no probabilístico. Muestra no probabilística o dirigida Subgrupo de la población en la que la elección de los elementos no depende de la probabilidad, sino de las características de la investigación.

El diseño de la investigación es descriptivo, según Tamayo (2004) es aquella que comprende la descripción, registro, análisis e interpretación de la naturaleza actual, composición o procesos de los fenómenos. El enfoque trabaja sobre las realidades de los hechos y sus características fundamentales, para presentarnos una interpretación correcta. ( $\mathrm{p}$. 54).

En la investigación se aplicaron la Encuesta. "Las encuestas consisten en un conjunto más o menos amplio de preguntas o cuestiones que se consideran relevantes para el rasgo, característica o variables que son objeto de estudio". (Bisquerra, 2009, p. 88). Básicamente, podemos decir que los dos pilares sobre las cuales se construye la encuesta son el marco teórico y los objetivos de la investigación. De esta manera, al construir la encuesta se toman en cuenta una serie de aspectos, ideas o creencias del investigador ante el problema a estudiar atendiendo a la revisión bibliográfica y otras investigaciones sobre el mismo tema, realizadas en otros contextos, así como los objetivos planteados en el estudio.

En los resultados de la investigación

Concepciones de la discriminación

\section{Orientación sexual e identidad de género diversas}


1. ¿Se puede obligar o convencer a una persona para que deje de ser LGTBI y sea heterosexual, o al revés?

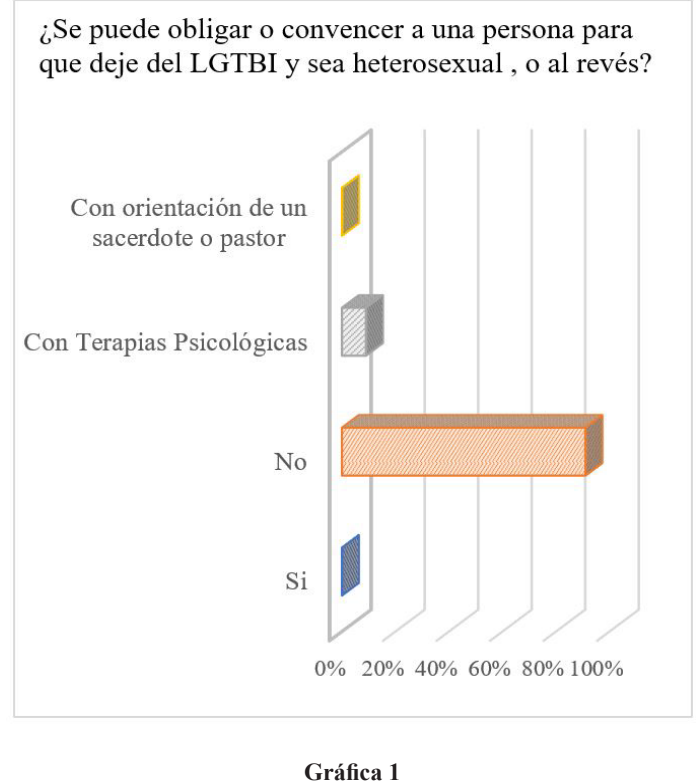

Fuentes: propias

Un 91\% de los encuestados, eligieron que no se puede obligar o convencer a una persona para que deje de ser LGTBI; seguidamente, responden un 9\% que si dejasen de pertenecer a la comunidad LGTBI a través de orientaciones o terapias psicológicas.

\section{2. ¿Qué sentimientos habitan en una persona LGTBI creyente}

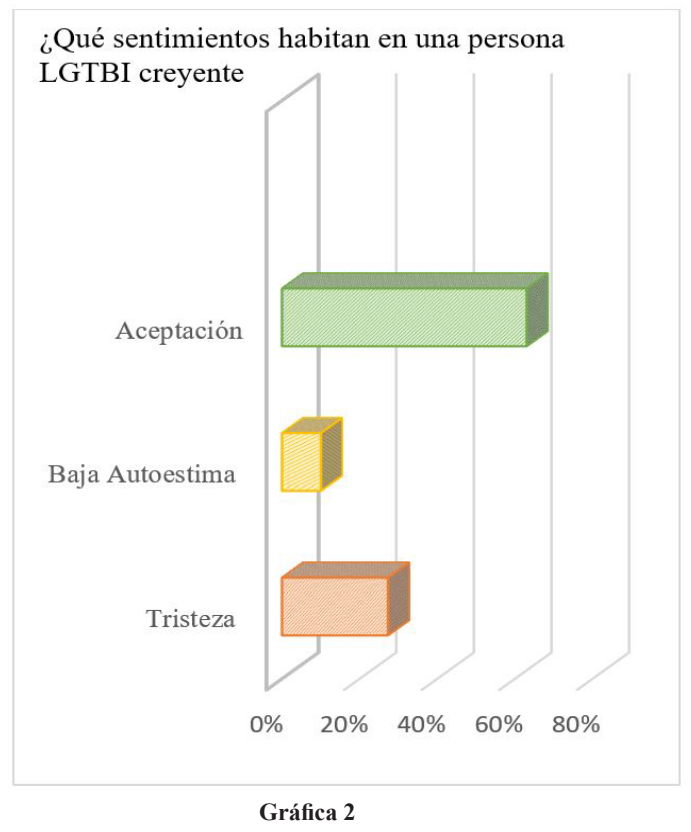

Fuentes: propias 
Un 63\% de los encuestado elijieron que el sentimiento que habitan en las persona LGTBI es la aceptación; en un segundo lugar, un $27 \%$ dicen que el sentimientos de tristeza se presenta en la comunidad LGTBI y por último, un $10 \%$ de los estudiantes encuestados presentan baja autoestima en las personas con LGTBI

3. Ha tenido o ha visto experiencia de rechazo o discriminación a causa de la expresión de la orientación sexual e identidad de género diversas en público

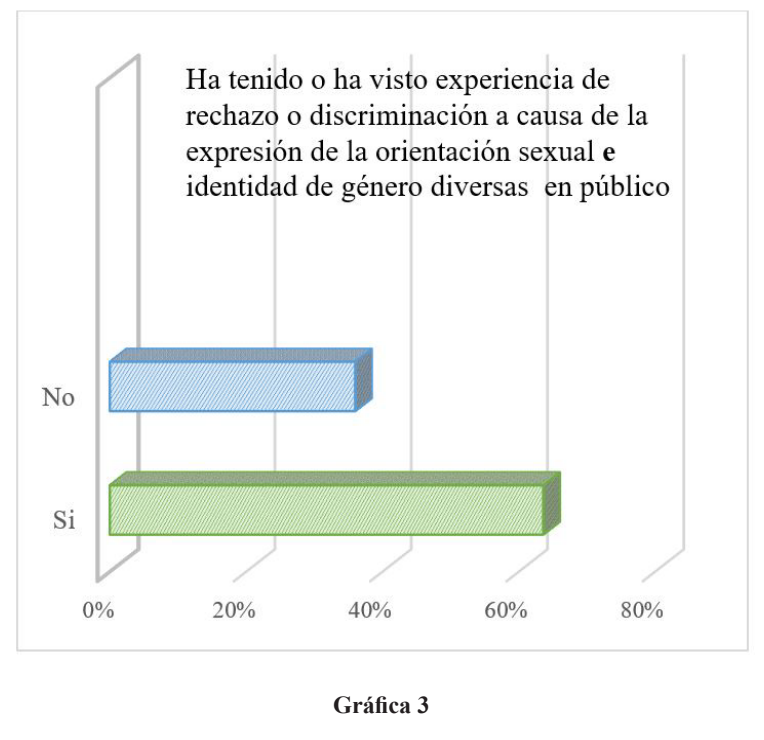

Fuentes: propias

Un $64 \%$ en los encuestados han visto rechazo o discriminación en la identidad de género diversas; un $36 \%$ en los encuestados no han tenido experiencia de rechazo o discriminación en la población LGTBI.

\section{Población de Negritudes}

4. Le produce asco las personas de color o población de Negritudes

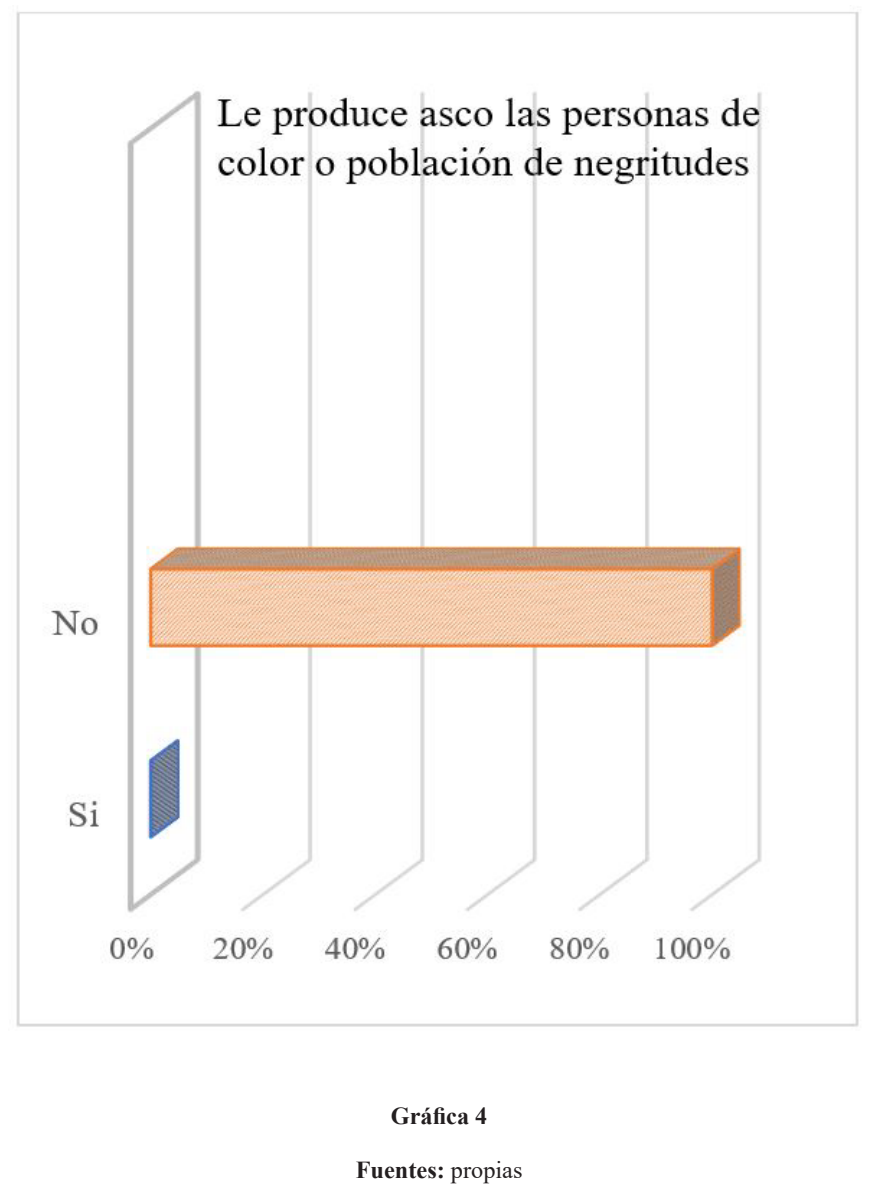

Un $100 \%$ en los encuestados no les produce asco las personas de color o la población de negritudes. 


\section{Las personas de color son discriminadas}

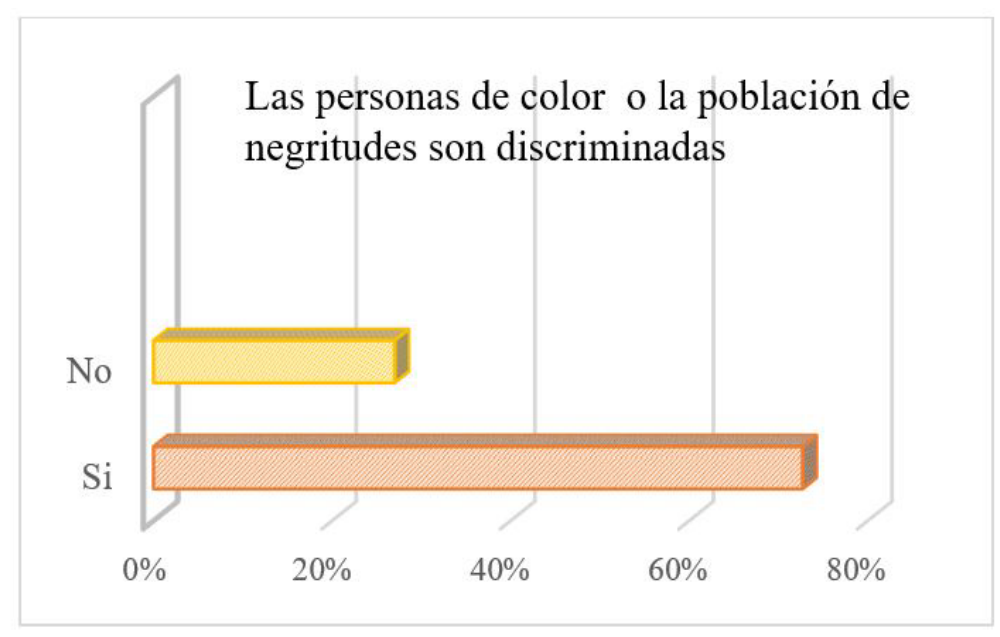

Gráfica 5

Fuentes: propias

Un $73 \%$ en los encuestados las personas de color o la población de negritudes, si son discriminadas y un $27 \%$ en los estudiantes encuestados seleccionaron la respuesta que no son discriminados.

\section{Hay oportunidad laboral para las personas de color o población de negritudes}

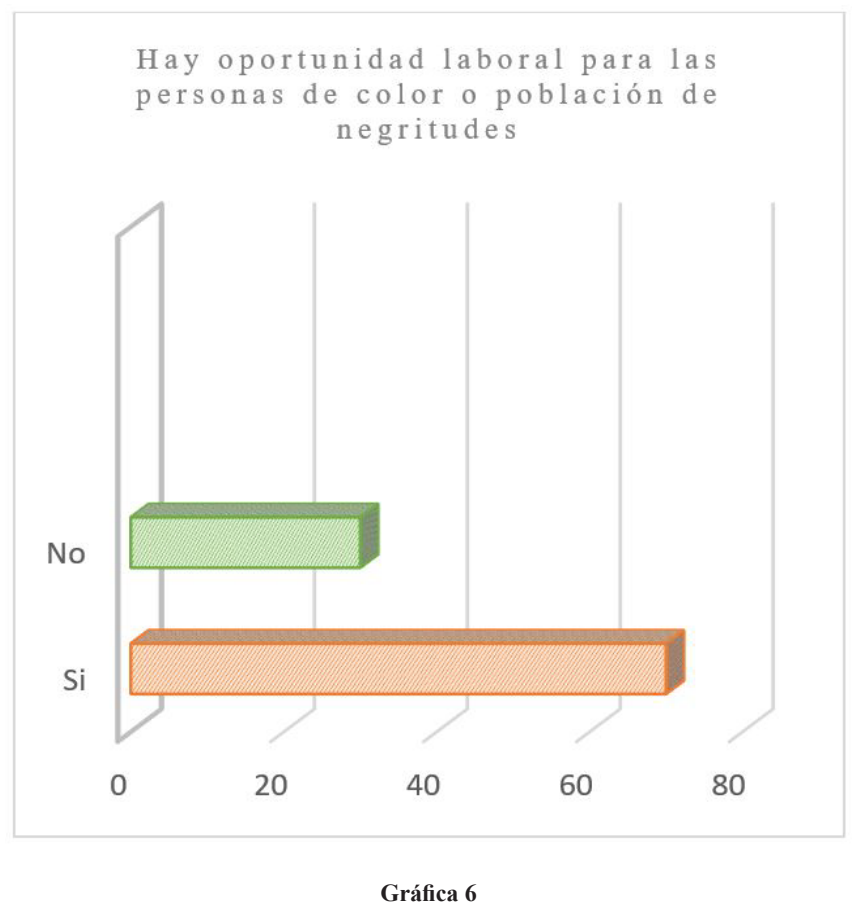

Fuentes: propias 
Un 70\% en los encuestados las personas de color o la población de negritudes, si tienen la oportunidad laboral y un $30 \%$ en los estudiantes encuestados seleccionaron la respuesta que no hay oportunidad laboral para la población de negritudes.

\section{Discusión}

Gómez Blanco, Carmen Ginary. (2018). En el diseño de una estrategia comunicativa para la inclusión social y el fomento de las prácticas culturales de la comunidad LGTBI en la universidad Francisco de Paula Santander Ocaña; se observó tres fases en donde se describen las actividades competentes para fomentar la inclusión social y las prácticas culturales de la comunidad LGBTI en la universidad Francisco de Paula Santander Ocaña. En la cual, se determinó que una de las causas principales que genera la exclusión social y la discriminación a los jóvenes que pertenecen a la comunidad LGTBI es la falta de información y de formación de los estamentos (estudiantes, docentes y administrativos) de la universidad Francisco de Paula Santander Ocaña en temas sobre inclusión social y demás subtemas que forman parte del enfoque de educación inclusiva.

Contreras-Manrique, R. de B., ContrerasManrique, L., Ovalle-Lizcano, T. V., EspinosaBohórquez, L. M., Valero-Bencardino, J., CoronelPeñuela, D. L., \& Cabrera-Reyes, A. (2020). Descripción de los problemas sociales influyentes en la transición moral en una sociedad cambiante a través del enfoque cuantitativo. El derecho ha tenido cambios históricos a través del tiempo, es inconcebible no pensar que el mismo llegue a una mutación por falta de condiciones en el futuro. Las diversas crisis por las que constantemente ha tratado de sobrevivir el planeta tales como la sobrepoblación, las pandemias que reducen la durabilidad, que inducirán al derecho a dar una vuelta y acoplarse a la civilización, llegará el tiempo, que el deber del estado ya no será asegurar la vida sino la supervivencia; en el caso del aborto, podría esta carencia de condiciones cambiar la opinión colectiva respecto a este tema de controversia mundial y pasar de ser una aberración a una solución eficaz para reducir la población, en consecuencia a un control de integrantes en la familia y la aprobación de la eutanasia como recurso que justifique una muerte digna, dejando lo ético y predomina lo conveniente; siendo la moral y el derecho piezas moldeables a las necesidades de la sociedad

Rodríguez Otero, Luis-Manuel, Ávila Zárate, Lydia del Carmen. (2019). Actitudes hacia la diversidad. La discriminación y el estigma en estudiantes de trabajo social mexicanos.

Socialmente ciertos rasgos o atributos son estructuralmente devaluados y generan procesos en los que se jerarquiza de forma inferior a quienes se etiquetan bajo dichas características, generando así procesos de violación de los derechos humanos, discriminación y exclusión social que son producidos, reproducidos por la sociedad y sus estructuras. Por lo tanto; en el proyecto, se observó, las siguientes expresiones actitudinales como la tranquilidad, el orgullo gay, la tolerancia hacia sus compañeros que no los comprende, el sentimiento de culpa por no cumplir una norma social, respeto mutuo, ser consciente, lealtad y libertad con respeto hacia la diferencia del otro.

\section{Conclusiones}

En la Universidad de Pamplona en el marco de la ruta por la acreditación institucional, inicia labores que logren generar un complemento en los procesos educativos, esto a través de la organización de espacios de capacitaciones que ayuden en la construcción de modelos adaptados en áreas de inclusión en beneficio a la comunidad LGBTI, negritudes, en general.

Así mismo se analizaron las percepciones de los estudiantes universitarios encuestados: 
Un mayor porcentaje de los estudiantes encuestados eligieron que no se puede obligar o convencer a una persona para que dejen la comunidad LGTBI; por lo tanto, aceptan la posición de los estudiantes que pertenecen a esta comunidad $\mathrm{y}$, por consiguiente, en un porcentaje mínimo, con sentimientos de tristeza y baja autoestima.

Un 64\% en los encuestados han visto rechazo o discriminación en la identidad de género diversas por parte de sus compañeros universitarios.

Un $73 \%$ en los encuestados, las personas de color o la población de negritudes, si son discriminadas.

En las oportunidades laborales, Un 70\% en los encuestados, eligieron que las personas de color o negritudes, si tienen oportunidad.

El papel de la comunidad educativa (padres y madres de familia, estudiantes, docentes, profesionales de psicología, enfermera, médico y trabajo social, es convivir diariamente con la diversidad de género y la comunidad negra con valores, en especial el respeto, los derechos humanos y dando cumplimiento a lo que establece la Constitución.

Es importante crear espacios de diálogo y convivencia métodos lúdicos para fomentar las relaciones sociales, la solidaridad y las diferencias en sus orientaciones sexuales en el campus universitario.

\section{Referencias}

Anselm, Strauss \& Juliet, Corbin. (2002). Bases de la investigación cualitativa. Técnicas y procedimientos para desarrollar la teoría fundamentada. Universidad de Antioquia. Colombia

Bazán, V. (2014). Control de las omisiones inconstitucionales e inconvencionales. Bogotá: Konrad Adenauer Stiftung (KAS)

Bisquerra, Rafael. et.al. (2009) Metodología de la investigación. Editorial Muralla, S.A. Madrid

Cáceres, M. O., Santiago, M. C., \& Rincón Leal, O. L. (2019). Emotional intelligence in the initial teacher training. Journal of Physics: Conference Series, 1329(1). doi:10.1088/17426596/1329/1/012012

Carrascal \& Prevert. (2012). La discriminación social desde una perspectiva psicosociológica. Antioquia, Colombia: revista de psicología universidad de Antioquia

Cestero Mancera, A. M. (2006). La comunicación no verbal y el estudio de su incidencia en fenómenos discursivos como la ironía. ELUA. Estudios de Lingüística, N. 20 (2006); pp. 57-77

Colombia, Corte Constitucional. (2014). Sentencia T-455 de 7 de julio de 2014. M. P. Luis Ernesto Vargas

Colombia, Corte Constitucional. (2014). Sentencia T-476 de 9 de julio de 2014. M. P. Alberto Rojas Colombia, Corte Constitucional. (2014). Sentencia T-970 de 15 de diciembre de 2014. M. P. Luis Ernesto Vargas

Constitución Política de Colombia. (2008). Artículo Constitucional del día, Colombia

Contreras-Manrique, R. de B.., ContrerasManrique, L.., Ovalle-Lizcano, T. V.., EspinosaBohórquez, L. M.., Valero-Bencardino, J.., Coronel-Peñuela, D. L.., \& Cabrera-Reyes, A... (2020). Descripción de los problemas sociales influyentes en la transición moral en una sociedad cambiante a través del enfoque cuantitativo. Eco Matemático, 11(2). https://doi. org/10.22463/17948231.3016

Corte Interamericana de Derechos Humanos. Cuadernillo de Jurisprudencia de la Corte Interamericana de Derechos 
Humanos. (2018). No. 19: Derechos de las personas LGTBI / Corte Interamericana

de Derechos Humanos. -- San José, C.R.: Corte IDH, 57 p.: $28 \times 22 \mathrm{~cm}$. ISBN digital 978-997736-240-3

El Universal. (2018). Sentencias de la corte constitucional sobre los derechos de la población LGTBI. Colombia

FRA- Agencia de los Derechos Fundamentales de la Unión Europea. (2009). Homofobia y discriminación por motivos de Orientación sexual e identidad de Género en los estados miembros de la UE

Gómez Blanco, C. G. (2018). Diseño de una estrategia comunicativa para la inclusión social y el fomento de las prácticas culturales de la comunidad LGTBI en la universidad Francisco de Paula Santander Ocaña

Guadarrama Zea, E. A. (2012). Discriminación por orientación sexual, resistencia desde la sociedad civil, México

Gutiérrez Solís, A. (2017). Estudian discriminación a la comunidad LGBT en universidades.

http://www.conacytprensa.mx/index.php/ciencia/ humanidades/16825-discriminacion-

comunidad-lgbt-universidades

Hernández, R; Fernández, C. y Baptista, P. (2014). Metodología de la Investigación. Bogotá: McGraw Hill. Bogotá: McGraw Hill. Sexta edición

Hurtado Caycedo, C.(2011). Modelo de doble tensión para analizar las estrategias de reconocimiento pleno de las diversidades sexuales en los debates de ciudadanía. ISSN-e 0717-2087, Nº 15, 2011, págs. 101-121

Jaramillo, J. E., Rincon Leal, J. F., \& Rincon
Leal, O. L. (2020). Social skills of physics Students in the mathematics degree program. Journal of Physics: Conference Series, 1674(1). doi:10.1088/1742-6596/1674/1/012017

Lamas Encabo, M (1947). Es una antropóloga mexicana, hija de padres argentinos, que se ha distinguido por su activismo como feminista

Lupicinio, I. (2008). Observación participante. Métodos cualitativos de investigación en ciencias sociales

Maroto, A. (2006). Reseña: Homosexualidad y Trabajo Social herramientas para la reflexión e intervención profesional. España

Mineducacion. (2016). Sanciones del manual de convivencia. Bogotá

Otero, L. M. R., \& Zárate, L. D. C. Á. (2019). Actitudes hacia la diversidad. La discriminación y el estigma en estudiantes de trabajo social mexicanos. Comunitaria: Revista internacional de trabajo y ciencias sociales, (18), 37-63

Parrado, M. H. (2012). ¿Qué es la hermenéutica? Universidad de la Salle

Penna Tosso, M. (2013). Formación inicial del profesorado para atender la diversidad afectivosexual: Una cuestión de derechos, salud mental y educación. Revista Iberoamericana de Educación edición digital, 66, 123-142

Penna Tosso, M. (2015). Homofobia en las aulas universitarias. Un metaanálisis. REDU. Revista de descendencia universitaria, REDU, ISSN-e 1887-4592, Vol. 13, No. 1.

Universidad Complutense de Madrid (España)

Psicólogos en Madrid EU. (2019). Teoría General de Sistemas de von Bertalanffy. Recuperado de: http://psicologosenmadrid.eu/teoria-general-de- 
sistemas-de-von-bertalanffy/

Quinche Ramírez, Manuel Fernando. (2016). Violencias, omisiones y estructuras que enfrentan las personas LGTBI. Universidad del Rosario, Colombia. Revista Estudios SocioJurídicos, vol. 18, no. 2, 2016

Ramírez Aristizábal, F. M. (2017). Inclusión educativa de estudiantes sexualmente diversos en Colombia periodo 2000 - 2015 -comprensiones pedagógicas de las sentencias de la corte constitucional. Bogotá, D.C. universidad abierta y a distancia - vuad

Ramírez Pavelic, M. (2013). La experiencia escolar en las narrativas de identidad sexual LGTB: un estudio fenomenológico retrospectivo. Universidad Autónoma de Madrid

Rey Sebastián, A. (2004). El derecho a la igualdad, las acciones positivas y el género. Buenos Aires

Rivera, D. C. L., \& de los Santos, P. J. (2017). Representaciones Sociales sobre las personas LGBTI en la universidad: perspectivas del profesorado y alumnado. Revista de Educación Inclusiva, 9(3)

Rodríguez Cepeda, J. (2005). Definición y concepto de la no discriminación. Universidad autónoma metropolitana- Azcapotzalco, distrito federal, México

Rodríguez-Otero, L. M., \& Facal-Fondo, T. (2019). Imaginarios, mitos y actitudes frente a la bisexualidad en estudiantes de Trabajo Social. Trabajo Social Global-Global Social Work, 9(17), 20-40

Rodríguez-Otero, L. M., \& Treviño-Martínez, L. (2016). Sexismo y actitudes hacia la homosexualidad, la bisexualidad y la transexualidad en estudiantes de Trabajo Social mexicanos. Trabajo Social Global-Global Social Work, 6(11), 3-30

Samaniego, J. \& Bermúdez, D. (2015). Discriminación hacia jóvenes Homosexuales en su entorno educativo. Cuenca, Ecuador: Universidad de cuenca Facultad de psicología

Shibley, J. (2006). Psicología y directora del Women's studies research Center de la Universidad de Wisconsi-Madison

Sierra, Á. (2009). Una aproximación a la teoría queer: el debate sobre la libertad y la ciudadanía. Cuadernos del Ateneo, 26, 29-42

Tamayo \& Tamayo. (2004). El Proceso de la Investigación. México: Limusa. p. 54

The Yogyakarta Principles on the Application of International Human Rights Law in Relation to Sexual Orientation and Gender Identity. Yogyakarta, 2007

Vadillo, M. N. (2018). Trato digno: concepto de la ley de defensa al consumidor y su aplicación

Zambrano Guerrero, C., Hernández Pasichaná, P., \& Guerrero Montero, A. (2019). Proceso de reconocimiento de la orientación sexual homosexual en estudiantes de una universidad pública: Proceso de reconocimiento de la orientación sexual homosexual en estudiantes de una universidad pública. Psicogente, 22(41), 1-29 